\title{
EL CURRICULUM UNIVERSITARIO EN TORNO A LA FORMACIÓN INTEGRAL
}

\author{
Silvia Morelli (Universidad Nacional de Rosario)* \\ silviatmorelli@hotmail.com
}

Recibido: 30/08/2013 Aceptado: 15/11/2013

\section{Resumen}

Este ensayo trata sobre las relaciones entre la formación integral y el currículum universitario desde una perspectiva posestructuralista. La Formación Integral no es una categoría nueva para la educación superior, pero cuando se la evoca se divisa un área variopinta que advierte un concepto complejo de abordar. Hace ya décadas que la universidad viene ocupándose de ofrecer a los estudiantes una formación que amplíe el horizonte de lo académico. Pretendiendo instalar el debate sobre esta categoría y arrojando ideas que aportan a su comprensión, se abordan cuestiones relativas a la formación integral como significante, al currículum universitario como un territorio para la formación integral y a la relación entre formación integral, responsabilidad social y democracia en la universidad.
\end{abstract}

\section{Palabras Claves}

Formación Integral - Currículum Universitario - Relación Universidad-Sociedad - Democracia - Significante Nodal.

\begin{abstract}
This paper discusses the relationship between the integral formation and university curriculum, through a poststructuralist perspective. Integral Formation is not a new category for higher education, but when it evokes we can see a varied area warning
\end{abstract}

* Magíster en Diseño de Estrategias de Comunicación. Facultad de Ciencia Política y Relaciones Internacionales de la Universidad Nacional de Rosario y Profesora en Ciencias de la Educación Facultad de Humanidades y Artes-UNR. Doctoranda en Humanidades y Artes con mención en Ciencias de la Educación UNR. Profesora Adjunta Área del Currículum de la Carrera de Ciencias de la Educación UNR. 
addressing a complex concept. For decades now the university has been seized to offer students training to expand the horizon of academics. Pretending to install the debate on this category and throwing ideas that contribute to its understanding, it addresses issues such as integral formation as a significant, the university curriculum as a territory for the integral formation and the relationship between the integral formation, social responsibility and democracy in the university.

\section{Key Words}

Integral Formation - University Curriculum - University-Society relationship Democracy - Nodal Significant.

\section{La Formación Integral como significante}

La formación integral puede ser entendida como significante nodal, según Laclau y Mouffe (2010: 152), un significante nodal es un punto discursivo que fija sentido en la cadena de significación en tanto significante privilegiado. La metáfora que mejor connota a los significantes nodales es la del capitoné, que entreteje su trama tensando los hilos de un tela con diferentes intensidades. En el derecho de la tela notamos los nudos que demuestran su fruncido. Esos nudos son los significantes nodales, nombres destacados en el discurso. En el revés de la trama se da lugar a los modos en los que se fabricaron las tensiones de los hilos. El revés muestra mucho más que el derecho acerca del tipo de trama a la que nos referimos. El derecho de la trama denota enunciación, como el ropaje que elegimos para decir, sin mostrar su revés, aunque lo contiene. El significante formación integral se constituye a partir de articulaciones que evocan principalmente al estudiante como sujeto de la pedagogía universitaria, a sus procesos cognitivos y poseedores de un saber experto. Pero además evoca a lo multidisciplinar, a las funciones académicas de la universidad, a la responsabilidad social de la universidad, a la ética de la formación, a la idea de futuro, como una alternativa a la crisis.

La flotación del significante formación integral lo convierte en un concepto ambiguo, polisémico, de esos conceptos que sirven para enunciar grandes acontecimientos pero no se dice mucho cada vez que se los nombra. Se presenta una tensión entre el significante y su significado, que en el caso de la formación integral deberíamos decir significados. Variados y diversos significados se ubican en un abanico teórico e ideológico tan amplio que cada vez que se la nombra entramos, sin excusas, en los juegos de lenguaje. Estos son el modo más sincero que encontró Wittgenstein (1953) para demostrar que no hay relación unívoca entre significado y significante, sino que el significado está determinado por el uso, por la forma más que por el nombre. Vuelvo al perturbador concepto de formación integral cuando encontramos muchos significados para un significante. 
Despejar hilos del capitoné, del nudo nocional de este significante ayudará a dar un sentido ético, político y pedagógico a la educación de los universitarios, a las funciones docentes y los sentidos de sus prácticas, a las tareas de la universidad como institución educativa y tal vez sea tiempo de volver a pensar tras el pretexto de la Formación Integral el sentido de la formación universitaria. Parafraseando a Derrida en aquello a lo que se refiere cuando llama la atención en la universidad sin condición.

La universidad moderna debería ser sin condición. Entendamos por «universidad moderna» aquella cuyo modelo europeo, tras una rica y compleja historia medieval, se ha tornado predominante, es decir "clásico», desde hace dos siglos, en unos Estados de tipo democrático. Dicha universidad exige y se le debería reconocer en principio, además de lo que se denomina la libertad académica, una libertad incondicional de cuestionamiento y de proposición, e incluso, más aún si cabe, el derecho de decir públicamente todo lo que exigen una investigación, un saber y un pensamiento de la verdad. Por enigmática que permanezca, la referencia a la verdad parece ser lo bastante fundamental como para encontrarse, junto con la luz (Lux), en las insignias simbólicas de más de una universidad... (Derrida, 1998: 2-3).

(...) Esta universidad sin condición no existe, de hecho, como demasiado bien sabemos. Pero, en principio y de acuerdo con su vocación declarada, en virtud de su esencia profesada, ésta debería seguir siendo un último lugar de resistencia crítica -y más que crítica- frente a todos los poderes de apropiación dogmáticos e injustos. (Derrida, 1998: 4).

Significantes como formación integral corren el riesgo de convertirse en aquellas categorías que contienen todo lo posible de ser explicado en la educación superior. Como si su carácter nodal lo habilitara a ocuparse de todo lo que amarra la noción integral. Algo similar ocurre cuando nos referimos a la complejidad, a lo transversal, a la flexibilidad. Hacemos jugar estos significantes como adjetivos y le damos carácter performativo cuando le pedimos acción y concreción de decisiones. Pero que la ambigüedad de la llamada formación integral no impida ser utilizada, no sea un límite para seguir pensando la educación superior como el horizonte que le da sentido a nuestras labores académicas y a fundar cada día como si fuese el primero, el compromiso con la sociedad.

El desafío consiste en no desconocer la característica ambivalente y flotante de la formación integral y aventurarse a tomarla para otorgarle significado que refleje las demandas locales, la cultura institucional, las características de los sujetos, las necesidades y contingencias que se advierten, el porvenir de universidad. Que esta tarea de conceptualización se impregne de reflexión, de juegos 
de lenguaje y de construcción de la identidad institucional. De este modo cada vez que se haga referencia a la formación integral se entra en un raro diálogo en el que no hace más que explicar qué se entiende por educación superior.

Reconocer las particularidades de la formación integral implica, en primera instancia despojarla de la significación vacía que intentan darle los documentos elaborados por organizamos internacionales o por instituciones ministeriales, dando el primer paso hacia la universidad sin condición a la que se refiere Derrida, cuando sugiere atribuirle a el lugar de resistencia crítica -y más que crítica- frente a todos los poderes de apropiación dogmáticos e injustos. Abrir el juego a la tensión entre lo vacío de las propuestas universales y arriesgarse a trabajar con las particularidades de las vidas universitarias como uno y sólo uno de los reflejos de la complejidad social.

\section{El currículum como territorio de aprendizajes, sujetos y calidad educativa}

La formación integral como problema educativo desembarca ineludiblemente en el territorio curricular y la lectura de esta cartografía se torna un problema de doble filo. Por un lado el currículum es uno de los espacios donde pueden materializarse las preocupaciones sobre la formación integral, materialización más que pertinente ya que éste es el dispositivo pedagógico que permite poner en lenguaje las intencionalidades político-educativas acerca de la formación universitaria.

Mientras que el otro lado del filo trae la exacerbación del territorio curricular. Posturas como estas consideran al currículum el único espacio para manifestar los debates sobre la formación integral; siendo que ésta es un problema de políticas de educación superior, de cultura universitaria, de responsabilidad académica, de legislación educativa y no sólo un problema curricular. Todas estas son expresiones cuyas aristas sobrepasan lo curricular, aunque no dejen de involucrarlo y aunque no deje de sostener que el currículum universitario es mucho más que el plan de estudios. En una lectura técnica, corremos el riesgo de reducir la formación integral a un asunto exclusivamente del currículum. Si verdaderamente fuera así, estamos ante un problema tanto para la noción de formación integral, que queda limitada a un solo plano, como también un problema para el currículum que lo dejamos en el lugar de un dispositivo de disciplinamiento. Por pretender darle toda la responsabilidad en el tratamiento de un problema educativo, empobrecemos al currículum en un reduccionismo técnico.

Decía entonces que el currículum es un dispositivo pedagógico que comunica las decisiones político-académicas, trazando trayectos de enseñanza y aprendizaje, de relación con la sociedad y la cultura, de proyección hacia los imaginarios futuros. Este dispositivo que se elabora en el marco de la cultura institucional, reconoce a la formación integral como un asunto educativo. 
¿Qué dilemas curriculares se advierten?, ¿qué conflictos se generan a la hora de optar por una formación integral?, ¿qué ocurre en las instituciones cuando incorporan en el currículum rasgos de formación integral? Decimos que existen dilemas curriculares cuando se generan posiciones encontradas que no permiten una salida de los asuntos académicos ni por el lado de la negociación, ni por el lado de la imposición. Dilemas cuando se instalan conflictos, aparentemente, irresolubles; decisiones sin opciones. ¿Cuáles es la más integral de las formaciones?, ¿Cuáles es la mejor formación integral?, ¿En qué se diferencian una formación integral de otras?, ¿Cuál es la dinámica y la regularidad de la formación integral en una universidad?

La incumbencia curricular de la formación integral se define en el proceso de sobredeterminación del curriculum (de Alba, 1991) como esa arena de lucha en la que se encuentran diferentes intereses, nociones, poderes. Referirse a la sobredeterminación implica distinguirla de la determinación, donde alguien toma la decisión y los demás la respetan y concretan. Una determinación curricular implica unidireccionalidad, ausencia de discusión de la idea, un determinismo de poder. Pasar de la determinación a la sobredeterminación requiere reconocer a los sujetos (en sentido diverso y colegiado); a las tensiones entre las decisiones político-educativas y a los debates que se plantean cuando se abre el juego a los diferentes grupos de decisión y poder. Se teje una trama que se apoya en la democracia para darle continuidad a la vida de la educación superior. Intento proponer a la sobredeterminación curricular como metodología política para la toma de decisiones en los asuntos del currículum, a través del lenguaje de la lucha colegiada, la organización sectorial o por claustros, la negociación o como sostiene Mouffe (2009:27), desde la lógica adversarial, en la que los otros son reconocidos como adversarios y no como enemigos.

La formación integral aparece vinculada con la calidad educativa, otro significante nodal que no desarrollo en este texto pero que lo traemos a cuento para cuestionar su presencia en la educación superior. Lejos de querer preguntar qué es la calidad educativa, porque no es el objeto que aquí convoca pregunto: ¿quién establece los criterios de calidad?, ¿para qué incorporar la calidad educativa en el currículum?, ¿qué grupos se benefician con la calidad educativa?, ¿qué relación existe entre la calidad educativa y el cambio educativo?, ¿es pertinente relacionar la calidad educativa con la formación integral?

En la compleja trama conceptual de la calidad educativa se presenta el aprendizaje de los estudiantes como uno de los conceptos que se le tornan problemáticos a la educación superior y que sea, tal vez, el que adquiere centralidad a la hora de definir el problema de la formación integral. Al ubicar al estudiante en este centro se subvierte el gráfico educativo en el que el docente y la enseñanza, o la tensión entre competencias y disciplinas, adquieren estatus como problemas en la educación superior. Cuando el problema es el estudiante, comienzan a tener lugar las alternativas pedagógicas. ¿Cómo pensar el porvenir 
del estudiante en contextos de crisis, de cambios de paradigma, de alteración de los valores, de redefinición de las instituciones, de surgimiento de nuevas prácticas y de múltiples alfabetizaciones? El mayúsculo desafío comienza a desenrollarse en el territorio del curriculum, como ese espacio en el que se definirán los presentes y futuros de la formación.

Múltiples prácticas, relacionadas con situaciones alternativas de aprendizaje pueden ser consideradas en el marco de una formación integral. Sería conveniente desprenderse de la lógica racionalista y suspender los universalismos para pensar el currículum de manera contextualizada y particularizada. Los enunciados universales, homogéneos, desbordantes de neutralidad, no pueden considerarse en un currículum universitario en tiempos de crisis. La tarea consiste en poner bajo sospecha todo aquello que es enunciado en términos generales y someterse, sin temores, ni prejuicios pero con decisión política y pedagógica, al armado de escenarios que reconozcan lo particular como el horizonte para el trabajo en torno al currículum. Aunque parezca una paradoja, lo particular como condición para lo integral.

\section{La Formación Integral, la responsabilidad social y la democracia}

Responsabilidad Social es otro de los conceptos instituidos en los últimos tiempos que puede ser entendido con abstracción si no se adecua a demandas concretas de los sujetos y las instituciones. El curriculum vuelve a ofrecerse como la arena en la que se expresan intenciones que tienden a la formación integral como la responsabilidad social, la ética profesional, la prospectiva sociocultural y multicultural. ¿Qué hacemos con estas categorías si derivan de la determinación de documentos curriculares, de la determinación hegemónica en el currículum universitario? Cuando el enunciado contiene la abstracción del vacío de lo universal, resulta engorroso inscribirlo en los juegos de lenguaje. Si se desechan las discusiones socio-políticas relacionadas con la democracia, entonces la formación integral merece ser revisada como concepto. Si no sirve para educar sujetos políticos socialmente responsables deberíamos replantear la función de la educación superior. Mouffe (2012:13) sostiene que la democracia liberal (como la que nos constituye como sociedad políticamente activa) se encuentra en una paradoja y argumenta que la incuestionada hegemonía del neoliberalismo representa una amenaza para las instituciones democráticas. La democracia liberal, fundada en la libertad y los derechos, cuestiona la soberanía popular cuando lo que se amenaza es la libertad.

En vez de tratar de borrar las huellas del poder y la exclusión, la política democrática nos exige que las pongamos en primer plano, de modo que sean visibles para adentrarse en el terreno de la disputa. $Y$ el hecho de que esto deba considerase como un proceso sin fin no debería ser causa de desesperación, ya que el deseo de alcanzar 
un destino final sólo puede concluir a la eliminación de lo político y la destrucción de la democracia. En una organización política democrática, los conflictos y las confrontaciones, lejos de ser un signo de imperfección, indican que la democracia está viva y se encuentra habitada por el pluralismo (Mouffe, 2012: 49-50).

La idea de democracia que está indicando Mouffe sostiene por un lado el agotamiento de la democracia moderna, de origen liberal y como contrapartida propone instalar una noción de democracia orientada hacia el reconocimiento de la soberanía popular y las particularidades del pluralismo. La formación integral de los estudiantes cobra sentido cuando se compromete directamente con la redefinición de la noción de democracia. Pero para ello existe otra tarea, la de tomar posición frente al escenario social y su relación con el resto de los escenarios. Porque si optamos por la definición de la globalización, por ejemplo, la relación inclusión-exclusión cambia notablemente. Es entonces cuando aparece el dilema curricular. ¿Cómo materializar la formación democrática en el currículum?, ¿Qué contenidos, qué prácticas, qué ejercicios?

\section{Una experiencia en proceso: Los Debates Emergentes}

Presentaré en este apartado un proyecto de investigación que se encuentra en pleno desarrollo (1). Tiende a estudiar las tareas del currículum universitario en la respuesta a las demandas sociales. Para ello se creó la categoría Debates Emergentes. A éstos se los entiende como aquellas problemáticas, discusiones y demandas culturales compartidas por los grupos sociales, que instalados como denuncia contingente, merecen ser considerados en la formación universitaria. Es así como pueden considerarse a la educación ambiental, las alfabetizaciones múltiples, la inclusión social, el multiculturalismo, los estudios de género, entre otros. Los procesos de sobredeterminación curricular y las políticas académicas tienen un grado de decisión importante en la inclusión de estas problemáticas en el currículum universitario.

Cabe destacar que su significación se articula con una reconstrucción semántica del campo del curriculum enmarcada en las complejas relaciones con la sociedad y la cultura. Los Debates Emergentes se ubican entre los problemas sociales y el saber académico. Es su caracterización problemática lo que genera nuestro interés para que se constituya en un objeto de análisis, transformando un problema social, de sentido común, en un problema académico, dándole entidad emergente.

A deferencia del discurso científico, la expresión Problema Social encuentra su sede habitual en la doxa, es decir, en lo que circula como creencia en los medios masivos de comunicación, en el discurso periodístico y en la vida cotidiana. Nos referimos al sentido 
repetido, al decir evidente, a lo que se da por sentado y se reitera de manera recurrente en el habla de una comunidad (Saur, 2011:76).

En lo que respecta a la constitución del sujeto, los Debates Emergentes surgen en el antagonismo social (Laclau, 1990), al impedirle a éste ser uno mismo. Es así como la problemática social emerge ante la denuncia de enajenación a los derechos sociales, civiles, ambientales, de alfabetización, etc. del sujeto. Tiende tanto a la exclusión de las identidades sociales y colectivas como de las identidades individuales. La decisión de educar en torno a estas problemáticas es porque lo que queda escindido y fuera de las consideraciones sociales es la propia condición de sujeto. La emergencia con la que estas coyunturas se constituyen problemáticas lleva inscripta el olvido del sujeto. Los Debates Emergentes en su carácter contingente, pertenecen a un sistema de opciones que fueron históricamente desechadas, negadas como problemáticas sociales y por lo tanto tratadas por fuera de los espacios curriculares. Al posicionarse en el plano académico, incorporándose al discurso universitario, toman cuerpo como significantes flotantes.

La flotación de significantes es una consecuencia de la arbitrariedad estructural del signo, donde un mismo significante puede ser vinculado a distintos significados en distintos contextos con sobredeterminaciones sociales, culturales, geográficas que impiden fijar estructuralmente un solo significado a la categoría de flotante, que nunca se completa de sentido de manera plena. (Laclau, 1990:44).

Los Debates Emergentes surgen en lo que de Alba llama La Crisis Estructural Generalizada (CEG).

Por ella entiende al debilitamiento general de los elementos de los sistemas relacionales de distintas estructuras interrelacionadas que a su vez conforman una estructura mayor y que definen las identidades de sus espacios social, político, cultural, etc. el cual conduce a la proliferación de elementos flotantes. Esto es, al debilitamiento de los elementos de las estructuras económicas, políticas, sociales, culturales, educativas, cognoscitivas, etc. Una crisis estructural generalizada se caracteriza por la desestructuración de las estructuras, más que por la estructuración de nuevas estructuras, aunque en su interior se producen fenómenos complejos de articulación, como los contornos sociales del mundo (de Alba, 2009:29-30).

La autora ubica a la CEG en la transición entre el siglo XX y el XXI y reconocerla en la desestructuración le permite identificarla en espacios amplios de tiempo que pueden posibilitar la constitución de nuevas estructuras, producidas de muy diversas maneras según las regiones y países o los grupos sociales. Una característica de esta desestructuración es que exige nuevas formas de 
pensar y actuar ensayando nuevas formas de vida y juegos de lenguaje $y$ cuando logran cierto grado de estructuración y articulación se reconocen como contornos sociales. Situaciones de CEG tienden a terminar cuando se configuran nuevas figuras de mundo. En la transición entre el siglo XX y el siglo XXI las situaciones de CEG se manifiestan a través de lo que la autora denomina los rasgos disruptivos, elementos que provienen de otras estructuras y elementos nuevos e inéditos. Estos tres componentes se articulan en los llamados contornos sociales, para ofrecer algún tipo de respuesta a la CEG. Esa capacidad de articulación se da en la medida en la que aparecen nombres que optamos por incluir en la serie de un significante inclusivo como Debates Emergentes.

Pretendemos inscribir este estudio en lo que los estudios genealógicos del curriculum. Un estudio de este tipo es un desafío intelectual en el que se ponen en juego las perspectivas posestructuralistas (Cherryholmes, 1999) posicionadas en las articulaciones entre el Análisis Político del Discurso y la Genealogía. El recorrido metodológico lleva al pasaje de los estudios genealógicos a la genealogía del curriculum. El plano de la filosofía para la construcción de esta metodología permite el reconocimiento de los sujetos pedagógicos, la cultura universitaria y sus relaciones con la sociedad y el poder como constitutivos en esta gramática curricular. Se hace visible el acontecimiento, que aparece donde menos se espera, para reconocerlo en las diferentes escenas curriculares. La genealogía permite construir discurso a través de las pequeñas apariencias. Se le otorga importancia a aquellos datos desconectados, que parecieran a simple vista, sin sentido. Implica seguir el hilo de la procedencia y buscar qué pasó con la dispersión, localizar accidentes, desviaciones. Incluyendo la clásica pregunta de la genealogía cuando interroga acerca de ¿cómo llegamos hasta aquí? El acontecimiento, la creatividad y la expresividad de los Debates Emergentes son registrados a través de la construcción de espacios biográficos y del reconocimiento de los discursos en sus superficies materiales, que no puede abstraerse de los escenarios históricos.

El Análisis Político de Discurso es una perspectiva que manifiesta el interés por la dimensión política de una significación, por las fijaciones parciales de los significados construidos en lo escrito, lo dicho, los actos, objetos y relaciones sociales. Buenfil Burgos (2012:56) sostiene que más que una teoría o una metodología, es una analítica u horizonte de intelección. En su configuración interdisciplinar involucra categorías provenientes de la filosofía, la teoría política, la semiología y el psicoanálisis y enfoques variados como el posestructuralismo, posmarxismo, hermenéutica y pragmatismo. Se construye sobre pilares conceptuales como los de discurso, hegemonía y subjetivación; consideradas por esta autora categorías fuertes de uso frecuente con implicaciones hacia otras como antagonismo y articulación (Laclau y Mouffe, 2010). Esta perspectiva nos permite analizar el campo del curriculum como una construcción discursiva políticamente involucrada. Es desde este enfoque que consideramos al curri- 
culum como pliegue de una trama textual configurada por las tensiones entre lo político, el conflicto y lo instituyente.

Con un estudio semejante pretendemos instalar no sólo nuevos modos de estudiar al curriculum universitario, sino además reconocerlo en su plena relación con la sociedad y los sentidos de la formación integral universitaria.

\section{Más allá de los discursos universales}

No cabe duda de que al tratar la formación integral de los estudiantes universitarios existe siempre una relación con el curriculum que no puede obviarse. Esta formación trae como marca de identidad el compromiso social de un sujeto socio-político, educado en clave democrática, cuestionando la racionalidad de la democracia liberal. Nos e trata de un problema de embaces y contenidos. Se trata de un concienzudo análisis social y político para restablecer las relaciones entre la universidad y la sociedad, reconociendo al curriculum como dispositivo político pedagógico involucrado en esa trama. En él, estudiante universitario tiene el doble lugar de sujeto y objeto del cambio social y esta identidad es la llave para su formación integral.

El sentido pedagógico de la formación integral plantea como requerimiento fundamental que sea una parte constitutiva de la educación universitaria. Estos requisitos se presentan a través de la consideración del curriculum como texto en su contexto (Lundgren, 1991; Beltrán Llavador, 2010). Vale decir que la gramática curricular carece de sentido si no es elaborada por los lenguajes y los usos que le otorgan los sujetos sociales, en la dimensión micro-política y cultural.

La tensión que se establece en el terreno curricular hace las veces de eslabón entre la universidad y la sociedad construyendo su propio lenguaje, que resiste a los enunciados universales de la educación superior. La tracción de esos enunciados hacia la micro-política institucional se convierte en una tarea imprescindible para reconfigurar la formación integral con sentido genuino. El horizonte se traza en torno a un sujeto político que cuestionará los escenarios de la democracia contemporánea ofreciendo otros significados a este significante flotante a través de los juegos políticos de lenguaje, al decir de Mouffe, que parafrasea al último Wittgenstein.

La tarea es precisa y el desafío, grande pero vale la pena tomar las riendas del curriculum y hacerlo jugar en el preciso espacio que a este le compete. Sin extralimitarlo a los asuntos de política universitaria, ni tampoco reducirlo a las meras discusiones de planes de estudio. Vale la pena tomar como eje central de este nuevo lenguaje a la formación integral del estudiante, pero ésta no será ciertamente integral si no se trastoca la naturaleza del proyecto democrático, que lejos del territorio moderno necesitará construir una nueva cartografía hacia la democracia agonista (Mouffe, 2009) que introduce la categoría de adversario como el sujeto de la pluralidad política, superando el modelo (moderno) de la democracia liberal y de la democracia deliberativa. Sin la reconfiguración del 
lugar del sujeto socio-político no podrán hacerse planteos sociales y culturales imprescindibles para diseñar y actuar en torno a la formación integral de los universitarios.

\section{Notas Bibliográficas}

(1) Me refiero a la investigación titulada "El curriculum universitario y las nuevas demandas sociales" (HUM 394) acreditado por la Secretaría de Ciencia y Tecnología de la Universidad Nacional de Rosario y radicado en el Instituto de Investigaciones de la Facultad de Humanidades y Artes. El equipo de investigadoras está integrado por Silvia Morelli como directora del proyecto y Marta Crivelli, Nora Mirna Smitt, María Florencia Bisignani, Érica Iturbe, Camila Carlachiani y Romina Craparo como integrantes. El mismo tiene una duración cuatrienal y se encuentra en su segundo año de implementación.

\section{Referencias Bibliográficas}

- Beltrán Llavador, F. (2010). Política, poder y control del curriculum. En Gimeno Sacristán, J. (comp.) Saberes e incertidumbres sobre el curriculum. (pp 47-64). Madrid: Morata.

- Buenfil Burgos, R. (2012). La teoría frente a las preguntas y el referente empírico en la investigación. En Jiménez, M. (coordinador). Investigación Educativa. Huellas metodológicas. (pp.51-71). México: Juan Pablos Editor.

- Cherryholmes, C. (1999). Poder y Crítica. México: Ediciones Pomares.

- De Alba, A. (2009). El curriculum universitario en el contexto de la Crisis Estructural Generalizada. En Orozco Fuentes, B. (Coordinadora). Curriculum: experiencias y configuraciones conceptuales en México (pp.27- 65). México: IISUE Educación-Plaza y Valdés Editores.

(2007). Curriculum-sociedad. El peso de la incertidumbre, la fuerza de la imaginación. México: IISUE Educación- Plaza y Valdés Editores. (1991). Evaluación curricular. Conformación conceptual del campo. Centro de Estudios sobre la universidad. México: Unidad Bibliográfica. Universidad Nacional Autónoma de México.

- Derrida, J. (1998). La universidad sin condición. En "Derrida en castellano". www.jaquesderrida.com.ar

- Laclau, E. (2008). La razón populista. Buenos Aires: Fondo de Cultura Económica.

- (1990). Nuevas reflexiones sobre la revolución de nuestro tiempo. Buenos Aires: Nueva Visión.

- Laclau, E. y Mouffe, C. (2010). Hegemonía y estrategia socialista. Argentina: Fondo de Cultura Económica.

- $\quad$ Lundgren, U. (1991). Teoría del curriculum y escolarización. Madrid: Morata.

- Mouffe, C. (2012). La paradoja democrática. Barcelona: Gedisa.

- - (2009). En torno a lo político. Argentina: Fondo de Cultura Económica.

- Saur, D. (2012). De la doxa al saber académico: El complejo pasaje del problema social al problema de la investigación. En Jiménez, M. (coordinador). Investigación Educativa. Huellas metodológicas (pp.73-93). México: Juan Pablos Editor.

- Wittgenstein, L. (1953). Investigaciones filosóficas. S/D. 